\title{
A CARTAN-TYPE RESULT FOR INVARIANT DISTANCES AND ONE-DIMENSIONAL HOLOMORPHIC RETRACTS
}

\author{
Colum Watt
}

\begin{abstract}
We derive conditions under which a holomorphic mapping of a taut Riemann surface must be an automorphism. This is an analogue involving invariant distances of a result of $\mathrm{H}$. Cartan. Using similar methods we prove an existence result for 1-dimensional holomorphic retracts in a taut complex manifold.
\end{abstract}

\section{Introduction}

In what follows, $W$ denotes a connected Riemann $\operatorname{surface}, \operatorname{Hol}(W, W)$ denotes the set of holomorphic self-maps of $W$ and $\operatorname{Aut}(W)$ denotes the set of biholomorphic maps of $W$ onto itself. A complex manifold $M$ is taut if and only if for each complex manifold $N$, each sequence of holomorphic mappings from $N$ to $M$ contains a subsequence which either converges uniformly on compact subsets of $N$ or is uniformly divergent to infinity (in the one point compactification of $M$ ) on compact subsets of $N$.

Definition 1.1. We call a distance function $d$ on a Riemann surface $W$ invariant if

$$
d(f(w), f(z)) \leq d(w, z) \quad \forall w, z \in W, \quad \forall f \in \operatorname{Hol}(W, W) .
$$

A Hermitian metric $h$ on $W$ is called invariant provided

$$
h\left(f_{*}(u), f_{*}(u)\right) \leq h(u, u) \quad \forall u \in \mathcal{O}_{w} W, \quad \forall w \in W, \quad \forall f \in \operatorname{Hol}(W, W) .
$$

We say that a distance function $d$ on $W$ is $\mathcal{C}^{k}(k \geq 1)$ if $d$ is the integrated distance function associated to a $\mathcal{C}^{k-1}$ Hermitian metric $h$ on $W$.

2000 Mathematics Subject Classification. 32F45, 32Q40, 53C60.

Key words. Taut complex manifold, invariant distance, automorphism, holomorphic retract. 
Remark 1. A standard example of an invariant metric is the square of the Kobayashi metric on a taut Riemann surface. For the unit disc in the complex plane, this metric is usually referred to as the Poincaré metric.

In his paper [4], J. P. Vigué proved the following result.

Theorem. Let $X$ and $W$ be connected, taut complex manifolds with $W$ 1-dimensional. Assume that $h$ is an invariant Hermitian metric on $W$ and choose $w \in W, x \in X$ and $v \in \mathcal{O}_{x} X \backslash\{0\}$. Then there exists a holomorphic retraction $\rho: X \rightarrow X$ such that $\rho(w)=x, \rho_{*}\left(\mathcal{O}_{w} W\right)=\mathbb{C} v$ and $\rho(X)$ is biholomorphic to $W$ if and only if $E(x, v)=F(x, v)$ (where $E, F: \mathcal{O} X \rightarrow \mathbb{R}^{+}$are invariant Finsler metrics which are defined in terms of $w, W, h, x$ and $X$ and which generalise the usual Carathéodory and Kobayashi metrics).

Key to his proof of this is the following result of H. Cartan [1].

Cartan's Theorem. Let $W$ be a taut Riemann surface. If $f \in \operatorname{Hol}(W, W)$ fixes some point $w \in W$ and has unimodular derivative at $w$ then $f \in$ $\operatorname{Aut}(W)$.

Note that the hypothesis of Cartan's result is equivalent to the requirement that $f$ fixes $w$ and that its derivative at $w$ is unitary with respect to every (in particular every invariant) Hermitian metric on $W$. In a remark in [4], J. P. Vigué defined the invariant pseudodistances $c_{X, x}^{W, w}$ and $k_{X, x}^{W, w}$ in terms of an invariant distance on $W$ (see Section 3 below). He would have liked to use these to investigate the existence of 1-dimensional holomorphic retracts through two given points of $X$. To do so, Vigué would have needed (but did not possess) an analogue of Cartan's result which uses an invariant distance in place of an invariant Hermitian metric. In Section 2 we prove such an analogue (Theorem 2.3) of Cartan's theorem under the assumption that the invariant distance arises from a continuous Hermitian metric on $W$. Then in the final section we use the invariant pseudodistances $c_{X, x}^{W, w}$ and $k_{X, x}^{W, w}$ (which generalise the usual Carathéodory distance and Kobayashi function on a complex manifold) and apply Theorem 2.3 to investigate the existence of holomorphic retractions of a complex manifold onto a 1-dimensional submanifold through two given points.

\section{Automorphisms of Riemann surfaces}

First we recall some standard notions from differential geometry. Let $h$ be a continuous Hermitian metric on a connected Riemann surface $W$. Thus $h$ determines a sesquilinear, positive definite inner product $h_{w}$ 
on each tangent space $\mathcal{O}_{w} W$ and $h_{w}$ varies continuously with $w$. The associated norm on $\mathcal{O}_{w} W$ is denoted $|\cdot|_{w}$ (to simplify notation, the subscript $w$ is often omitted). If $f \in \operatorname{Hol}(W, W)$ we denote its derivative at $w$ by

$$
f_{* w}: \mathcal{O}_{w} W \rightarrow \mathcal{O}_{f(w)} W .
$$

The operator norm of $f_{* w}$ (with respect to $|\cdot|_{w}$ and $|\cdot|_{f(w)}$ ) is denoted $\left\|f_{* w}\right\|$ (or by $\left\|f_{*}\right\|$ when $w$ is clear from the context). As $\mathcal{O}_{w} W$ is onedimensional it follows that

$$
\left|f_{* w}(u)\right|_{f(w)}=|| f_{* w}|| \cdot|u|_{w} \quad \forall u \in \mathcal{O}_{w} W .
$$

Continuity of $h$ implies that the map $w \mapsto\left\|f_{* w}\right\|$ is continuous. A piecewise $\mathcal{C}^{1}$ path in $W$ is a mapping $\gamma:[a, b] \rightarrow W$ for which there exists a finite set of points $a=t_{0}<t_{1}<\cdots<t_{n}=b$ such that $\left.\gamma\right|_{\left[t_{i}, t_{i+1}\right]}$ is $\mathcal{C}^{1}$ and has nowhere vanishing tangent for each $i=0, \ldots, n-1$. The length of such a path $\gamma$ is defined by

$$
l(\gamma)=\int_{a}^{b} h\left(\gamma^{\prime}(t), \gamma^{\prime}(t)\right)^{\frac{1}{2}} d t=\int_{a}^{b}\left|\gamma^{\prime}(t)\right| d t .
$$

For any two points $w$ and $z$ in $W$ the distance $d(w, z)$ is defined by

$$
d(w, z)=\inf \left\{l(\gamma): \gamma \text { is a piecewise } \mathcal{C}^{1} \text { path from } w \text { to } z\right\} .
$$

This function is clearly symmetric, positive and satisfies the triangle inequality. It is a standard result that $d(w, z)>0$ when $w \neq z$ and that $d$ generates the given topology on $W$ (for example, see [2]). The open ball $B(w, r) \subset W$ with centre $w$ and radius $r>0$ is given by

$$
B(w, r)=\{z \in W: d(w, z)<r\} .
$$

If $d$ is the distance arising from a hermitian metric $h$, it is easy to show that invariance of $h$ implies invariance of $d$. In this first proposition we prove a converse result.

Proposition 2.1. Let $d$ be the integrated distance associated to a continuous Hermitian metric $h$ on a Riemann surface $W$. If $d$ is invariant then $h$ must also be invariant.

Proof: Assume that there exists $f \in \operatorname{Hol}(W, W)$ such that $\left\|f_{* w}\right\|>1$ for some $w \in W$. We will show that $d$ cannot be invariant.

As $\left\|f_{*}\right\| \neq 0$ at $w, f$ maps some neighbourhood $U$ of $W$ biholomorphically onto an open neighbourhood of $f(w)$. Shrinking $U$ if necessary and using continuity, we may assume that $\left\|f_{*}\right\| \geq 1+\epsilon$ on $U$ for some $\epsilon>0$. 
Choose $\delta>0$ such that $B(f(w), \delta) \subset f(U)$. Let $y \in B(f(w), \delta)$ and let $\gamma$ be a path from $f(w)$ to $y$ such that

$$
d(f(w), y) \leq l(\gamma)<\delta .
$$

As any path which leaves $B(f(w), \delta)$ will have length at least $\delta$, the image of $\gamma$ must be contained in $B(f(w), \delta)$. Thus we may write $\gamma=f \sigma$ where $\sigma=\left(\left.f\right|_{U}\right)^{-1} \gamma$ lies in $U$ and starts at $w$. Denote the endpoint $\left(\left.f\right|_{U}\right)^{-1}(y)$ of $\sigma$ by $z$. Then

$$
\begin{aligned}
l(\gamma) & =\int\left|\gamma^{\prime}(t)\right| d t \\
& =\int\left|(f \sigma)^{\prime}(t)\right| d t \\
& =\int|| f_{* \sigma(t)}|| \cdot\left|\sigma^{\prime}(t)\right| d t \\
& \geq \int(1+\epsilon)\left|\sigma^{\prime}(t)\right| d t \\
& \geq(1+\epsilon) d(w, z) .
\end{aligned}
$$

Taking the infimum over paths joining $f(w)$ to $y=f(z)$, it follows that

$$
d(f(w), f(z)) \geq(1+\epsilon) d(w, z) .
$$

Hence $d$ cannot be invariant.

Proposition 2.2. Let $d$ be an invariant $\mathcal{C}^{1}$ distance on a Riemann surface $W$. Let $f \in \operatorname{Hol}(W, W)$ and assume that there exists a sequence of paths $\gamma_{n}:\left[0, a_{n}\right] \rightarrow W$ which all start at $w$ and for which

$$
\lim _{n \rightarrow \infty} l\left(\gamma_{n}\right)=\lim _{n \rightarrow \infty} l\left(f \gamma_{n}\right)=a>0 .
$$

Then $\left\|f_{* w}\right\|=1$.

Proof: Invariance of $d$ implies that $\left\|f_{*}\right\| \leq 1$ everywhere. Assume that $\left\|f_{* w}\right\|=r<1$. As $d$ is $\mathcal{C}^{1}, w \mapsto\left\|f_{* w}\right\|$ is continuous and hence there exists $\epsilon>0$ such that $\left\|f_{*}\right\|<\frac{1+r}{2}$ on $B(w, \epsilon)$. For each $n$ define $t_{n} \in$ $\left(0, a_{n}\right]$ by

$$
t_{n}= \begin{cases}a_{n} & \text { if } \gamma_{n}\left(\left[0, a_{n}\right]\right) \subset B(w, \epsilon) \\ \sup \left\{t: \gamma_{n}([0, t]) \subset B(w, \epsilon)\right\} & \text { otherwise. }\end{cases}
$$


Then

$$
\begin{aligned}
l\left(\left.f \gamma_{n}\right|_{\left[0, t_{n}\right]}\right) & =\int_{0}^{t_{n}}\left|\left(f \gamma_{n}\right)^{\prime}(t)\right| d t \\
& =\int_{0}^{t_{n}}|| f_{* \gamma_{n}(t)}|| \cdot\left|\gamma_{n}^{\prime}(t)\right| d t \\
& <\frac{1+r}{2} \int_{0}^{t_{n}}\left|\gamma_{n}^{\prime}(t)\right| d t \\
& =\frac{1+r}{2} l\left(\left.\gamma_{n}\right|_{\left[0, t_{n}\right]}\right)
\end{aligned}
$$

and hence

$$
\begin{aligned}
l\left(f \gamma_{n}\right) & =l\left(\left.f \gamma_{n}\right|_{\left[0, t_{n}\right]}\right)+l\left(\left.f \gamma_{n}\right|_{\left[t_{n}, a_{n}\right]}\right) \\
& <\frac{1+r}{2} l\left(\left.\gamma_{n}\right|_{\left[0, t_{n}\right]}\right)+l\left(\left.\gamma_{n}\right|_{\left[t_{n}, a_{n}\right]}\right) \\
& =l\left(\gamma_{n}\right)-\frac{1-r}{2} l\left(\left.\gamma_{n}\right|_{\left[0, t_{n}\right]}\right) \\
& \leq l\left(\gamma_{n}\right)-\frac{1-r}{2} \min \left(\epsilon, l\left(\gamma_{n}\right)\right) \quad \forall n .
\end{aligned}
$$

Thus

$$
\lim _{n \rightarrow \infty} l\left(f \gamma_{n}\right) \leq a-\frac{1-r}{2} \min (\epsilon, a)<a \quad \text { since } a>0
$$

As this contradicts the hypothesis that $l\left(f \gamma_{n}\right)$ converges to $a$, our assumption that $\left\|f_{* w}\right\|<1$ must have been false.

We combine these two propositions to prove the following theorem.

Theorem 2.3. Let $d$ be a $\mathcal{C}^{1}$ invariant distance on a taut Riemann surface $W$. Assume that $f \in \operatorname{Hol}(W, W)$ and that there are distinct points $w$ and $z$ in $W$ satisfying

$$
f(w)=w \text { and } d(w, z)=d(w, f(z)) .
$$

Then $f \in \operatorname{Aut}(W)$.

Proof: Let $\gamma_{n}$ be a sequence of paths from $w$ to $z$ whose lengths converge to $d(w, z)$. Taking the limit as $n \rightarrow \infty$ in the inequality

$$
l\left(\gamma_{n}\right) \geq l\left(f \gamma_{n}\right) \geq d(f(w), f(z))=d(w, z)
$$

we deduce that

$$
\lim _{n \rightarrow \infty} l\left(\gamma_{n}\right)=\lim _{n \rightarrow \infty} l\left(f \gamma_{n}\right)=d(w, z)>0
$$


Proposition 2.2 now implies that $\left\|f_{* w}\right\|=1$. Since $w$ is fixed by $f$ and $\mathcal{O}_{w}(W)$ is one dimensional, $f_{* w}$ must be given by multiplication by a unimodular complex number. Cartan's theorem now implies that $f \in \operatorname{Aut}(W)$.

Corollary 2.4. Let $W$ be a taut Riemann surface and suppose $f \in$ $\operatorname{Hol}(W, W)$ fixes two distinct points of $W$. Then $f \in \operatorname{Aut}(W)$.

Proof: The map $f$ preserves the Kobayashi distance between the two fixed points. As the Kobayashi distance is $\mathcal{C}^{\infty}$ (for any taut Riemann surface) the preceding theorem implies that $f \in \operatorname{Aut}(W)$.

Corollary 2.5. If $f \in \operatorname{Hol}(W, W)$ fixes two distinct points $w, z \in W$ which can be joined by a unique path $\gamma:[0, a] \rightarrow W$ satisfying

$$
d(w, z)=l(\gamma) \text { and } l\left(\left.\gamma\right|_{[0, t]}\right)=t \quad \forall t
$$

then $f$ is the identity map.

Proof: The path $f \gamma$ also joins $w$ to $z$. For any $t \in[0, a]$ we have

$$
\begin{aligned}
d(w, z) & =l\left(\left.\gamma\right|_{[0, t]}\right)+l\left(\left.\gamma\right|_{[t, a]}\right) \\
& \geq l\left(\left.f \gamma\right|_{[0, t]}\right)+l\left(\left.f \gamma\right|_{[t, a]}\right) \quad \text { by invariance } \\
& \geq d(f(w), f(z)) \\
& =d(w, z) .
\end{aligned}
$$

It follows that we must have $l\left(\left.\gamma\right|_{[0, t]}\right)=l\left(\left.f \gamma\right|_{[0, t]}\right)$ for all $t$. Our uniqueness hypothesis for $\gamma$ now implies that $f(\gamma(t))=\gamma(t)$ for all $t$, so $f$ fixes each point on $\gamma([0, a])$. The identity theorem for analytic functions implies that $f$ is the identity map.

Remark 2. If the distance $d$ is $\mathcal{C}^{4}$ then each point $w \in W$ has a neighbourhood $U$ such that any two points of $U$ can be joined by a unique path in $U$ which satisfies the hypothesis of $\gamma$ in the preceding corollary (such paths are usually called length minimising geodesics). For a taut Riemann surface $W$, the Kobayashi distance is $\mathcal{C}^{\infty}$ and hence any holomorphic map $f \in \operatorname{Hol}(W, W)$ which fixes two sufficiently close points must be the identity mapping.

Remark 3. The biholomorphism $w \mapsto \frac{1}{w}$ on the annulus $A=\{w \in \mathbb{C}$ : $\left.\frac{1}{2}<|w|<2\right\}$ fixes the two points 1 and -1 . However there is more than one length minimising geodesic joining these two points in $A$ (with respect to the Kobayashi metric). 


\section{One-dimensional holomorphic retracts}

In this section, following J. P. Vigué [4], we define analogues of the Carathéodory pseudodistance and the Kobayashi function on a complex manifold. We use these to examine the existence of holomorphic retractions of a complex manifold onto a 1-dimensional complex submanifold through two given points.

Let $x_{1}, x_{2}, \ldots, x_{n}$ and $y_{1}, y_{2}, \ldots, y_{n}$ be ordered sequences of points in the complex manifolds $X$ and in $Y$ respectively. Then

$$
\begin{aligned}
\operatorname{Hol}\left(X, x_{1}, \ldots, x_{n}, Y, y_{1}, \ldots, y_{n}\right) & \\
& =\left\{f \in \operatorname{Hol}(X, Y): f\left(x_{i}\right)=y_{i} \quad \forall i=1, \ldots, n\right\} .
\end{aligned}
$$

Let $W$ be a connected Riemann surface and $d$ an invariant distance on $W$. Fix a point $w \in W$ and let $X$ be a complex manifold with basepoint $x \in X$. Then we define a Carathéodory type function on $X \times X$ with values in $[0, \infty]$ by

$c_{X, x}^{W, w}\left(x_{1}, x_{2}\right)=\sup \left\{d\left(f\left(x_{1}\right), f\left(x_{2}\right)\right): f \in \operatorname{Hol}(X, x, W, w)\right\} \forall x_{1}, x_{2} \in X$.

The Kobayashi version $k_{X, x}^{W, w}\left(x_{1}, x_{2}\right)$ is defined as follows

(i) If $\operatorname{Hol}\left(W, w, w_{1}, w_{2}, X, x, x_{1}, x_{2}\right)=\emptyset$ for all $w_{1}$ and $w_{2}$ in $W$ then

$$
k_{X, x}^{W, w}\left(x_{1}, x_{2}\right)=\infty .
$$

(ii) Otherwise $k_{X, x}^{W, w}\left(x_{1}, x_{2}\right)$ is given by

$$
\inf \left\{d\left(w_{1}, w_{2}\right): w_{1}, w_{2} \in W, f \in \operatorname{Hol}\left(W, w, w_{1}, w_{2}, X, x, x_{1}, x_{2}\right)\right\} .
$$

It follows from the invariance of $d$ that

$$
c_{X, x}^{W, w}\left(x_{1}, x_{2}\right) \leq k_{X, x}^{W, w}\left(x_{1}, x_{2}\right) \quad \forall x_{1}, x_{2} .
$$

For the special case $(X, x)=(W, w)$, the invariance of $d$ also implies

$$
c_{W, w}^{W, w}\left(w_{1}, w_{2}\right)=k_{W, w}^{W, w}\left(w_{1}, w_{2}\right)=d\left(w_{1}, w_{2}\right) \quad \forall w_{1}, w_{2} \in W .
$$

As in the cases of the usual Carathéodory and Kobayashi functions it is straightforward to show that for all $f \in \operatorname{Hol}(X, x, Y, y)$ and $x_{1}, x_{2} \in X$

$$
c_{X, x}^{W, w}\left(x_{1}, x_{2}\right) \geq c_{Y, y}^{W, w}\left(f\left(x_{1}\right), f\left(x_{2}\right)\right)
$$

and

$$
k_{X, x}^{W, w}\left(x_{1}, x_{2}\right) \geq k_{Y, y}^{W, w}\left(f\left(x_{1}\right), f\left(x_{2}\right)\right) .
$$

If we take the usual Kobayashi distance on $W$ as our invariant distance $d$, then it is easy to see that the resulting function $k_{X, x}^{W, w}$ satisfies

$$
k_{X, x}^{W, w} \leq k
$$


where $k$ denotes the usual Kobayashi function given by

$$
\begin{aligned}
k\left(x, x_{1}\right)=\inf \left\{\tanh ^{-1}\left|\frac{z-w}{1-\bar{w} z}\right|:\right. & \exists w, z \in \mathbb{D} \\
& \text { with } \left.\operatorname{Hol}\left(\mathbb{D}, w, z, X, x, x_{1}\right) \neq \emptyset\right\}
\end{aligned}
$$

where $\mathbb{D}$ denotes the unit disc in the complex plane.

We now use the functions $c_{X, x}^{W, w}$ and $k_{X, x}^{W, w}$ to give a criterion for deciding when there exists a holomorphic retraction of a complex manifold $X$ onto a submanifold biholomorphic to $W$ which passes through two given points of $X$. First we recall the definition of a holomorphic retract.

Definition 3.1. A holomorphic retraction of $X$ is a holomorphic mapping $\rho: X \rightarrow X$ such that

$$
\left.\rho\right|_{\rho(X)} \text { is the identity map on } \rho(X) .
$$

The set $\rho(X)$ is called a holomorphic retract of $X$. It is closed and analytic.

Proposition 3.2. Let $x$ and $x_{1}$ be distinct points in a complex manifold $X$ and let $d$ be an invariant distance on a Riemann surface $W$. Assume that there exists a holomorphic retraction $\rho: X \rightarrow X$ such that $x, x_{1} \in \rho(X)$ and $\rho(X)$ is biholomorphic to $W$. Then there exists some point $w \in W$ for which

$$
0<c_{X, x}^{W, w}\left(x, x_{1}\right)=k_{X, x}^{W, w}\left(x, x_{1}\right)<\infty .
$$

Proof: Let $i: W \rightarrow X$ be a biholomorphism of $W$ onto $\rho(X)$. Put $w=$ $i^{-1}(x)$ and $w_{1}=i^{-1}\left(x_{1}\right)$. The three inequalities

(i) $d\left(w, w_{1}\right) \geq k_{X, x}^{W, w}\left(i(w), i\left(w_{1}\right)\right)=k_{X, x}^{W, w}\left(x, x_{1}\right)$

(ii) $c_{X, x}^{W, w}\left(x, x_{1}\right) \geq d\left(i^{-1} \rho(x), i^{-1} \rho\left(x_{1}\right)\right)=d\left(w, w_{1}\right)$

(iii) $c_{X, x}^{W, w}\left(x, x_{1}\right) \leq k_{X, x}^{W, w}\left(x, x_{1}\right)$

combine to give

$$
0<d\left(w, w_{1}\right) \leq c_{X, x}^{W, w}\left(x, x_{1}\right) \leq k_{X, x}^{W, w}\left(x, x_{1}\right) \leq d\left(w, w_{1}\right)<\infty
$$

since $w \neq w_{1}$. The result follows.

By strengthening our hypotheses, we can prove the following converse to this proposition. 
Theorem 3.3. Let $x$ and $x_{1}$ be distinct points in a connected, taut complex manifold $X$. Let $d$ be a $\mathcal{C}^{1}$ invariant distance on a taut Riemann surface $W$. If there is some point $w \in W$ for which

(a) $c_{X, x}^{W, w}\left(x, x_{1}\right)=k_{X, x}^{W, w}\left(x, x_{1}\right)<\infty$ and

(b) the open ball $B(w, r)$ has compact closure in $W$ (where $\left.r=k_{X, x}^{W, w}\left(x, x_{1}\right)\right)$, then there exists a holomorphic retraction $\rho: X \rightarrow X$ such that $x, x_{1} \in$ $\rho(X)$ and $\rho(X)$ is biholomorphic to $W$.

Proof: Assume that there is a point $w \in W$ which satisfies the hypotheses (a) and (b). By tautness of $X$ we can find maps $f, f_{1}, f_{2}, \ldots$ in $\operatorname{Hol}(W, w, X, x)$ and a sequence of points $z_{n} \in W$ such that

(i) $f_{n} \rightarrow f$ uniformly on compact sets,

(ii) $f_{n}\left(z_{n}\right)=x_{1}$ for each $n$,

(iii) $\lim _{n \rightarrow \infty} d\left(w, z_{n}\right)=k_{X, x}^{W, w}\left(x, x_{1}\right)$.

As $\overline{B(w, r)}$ is compact and $W$ is locally compact, there exists $\epsilon>0$ such that $\overline{B(w, r+\epsilon)}$ is compact. By (iii), there exists $N$ such that $z_{n} \in$ $B(w, r+\epsilon)$ for all $n \geq N$. Compactness of $\overline{B(w, r+\epsilon)}$ implies that $z_{n}$ has a convergent subsequence. Passing to this subsequence if necessary, we may assume that $z_{n}$ converges to $z$ (say). Since $d$ is continuous, we obtain

$$
d(w, z)=\lim _{n \rightarrow \infty} d\left(w, z_{n}\right)=k_{X, x}^{W, w}\left(x, x_{1}\right) .
$$

As the set $\left\{z, z_{1}, z_{2}, \ldots\right\}$ is compact, conditions (i) and (ii) imply that

$$
f(z)=\lim _{n \rightarrow \infty} f_{n}\left(z_{n}\right)=x_{1} .
$$

Note that $z$ and $w$ are distinct. Otherwise we would would have $x=$ $f(w)=f(z)=x_{1}$ which contradicts the hypothesis that $x$ and $x_{1}$ are distinct.

Next we use the tautness of $W$ to construct a sequence $g_{n} \in$ $\operatorname{Hol}(X, x, W, w)$ which converges uniformly on compact sets (to $g$ say) such that

$$
\lim _{n \rightarrow \infty} d\left(g_{n}(x), g_{n}\left(x_{1}\right)\right)=c_{X, x}^{W, w}\left(x, x_{1}\right) .
$$

Let $w_{1}=g\left(x_{1}\right)$. As $\left(g_{n}(x), g_{n}\left(x_{1}\right)\right)$ converges to $\left(g(x), g\left(x_{1}\right)\right)=\left(w, w_{1}\right)$ and $d$ is continuous, we obtain

$$
d\left(w, w_{1}\right)=c_{X, x}^{W, w}\left(x, x_{1}\right) .
$$


Since $g(f(w))=w$ and $g(f(z))=w_{1}$, equations (1) and (2) yield $d(w, z)=d(w, g(f(z))$. As $w \neq z$, Theorem 2.3 implies that $g f \in$ $\operatorname{Aut}(W)$. Now set $\theta=(g f)^{-1}$ and define $\rho: X \rightarrow X$ by

$$
\rho=f \theta g .
$$

It is easy to verify that $\rho$ is a holomorphic retraction and that $g$ maps $\rho(X)$ biholomorphically onto $W$.

Corollary 3.4. Let $x$ and $x_{1}$ be distinct points in a connected, taut complex manifold $X$. Let d be a complete, invariant, $\mathcal{C}^{1}$ distance on a taut Riemann surface $W$. If there is some point $w \in W$ for which

$$
c_{X, x}^{W, w}\left(x, x_{1}\right)=k_{X, x}^{W, w}\left(x, x_{1}\right)<\infty
$$

then there exists a holomorphic retraction $\rho: X \rightarrow X$ such that $x, x_{1} \in$ $\rho(X)$ and $\rho(X)$ is biholomorphic to $W$.

Proof: As $W$ is locally compact and $d$ is complete and inner, the HopfRinow theorem (see $[\mathbf{3}])$ implies that each open ball $B(w, s)(s>0)$ has compact closure. Thus all of the hypotheses of the previous theorem are satisfied and the corollary follows.

Remark 4. The Kobayashi distance for a taut Riemann surface $W$ is both $\mathcal{C}^{\infty}$ and complete and thus may validly be used in applying the preceding corollary. However, for explicit calculation it may be simpler to use an invariant distance on $W$ other than Kobayashi's.

Remark 5. The proof of Theorem 3.3 implies that $k_{X, x}^{W, w}\left(x, x_{1}\right)>0$. In fact, it is not difficult to show that if $x$ and $x_{1}$ are distinct points in a taut complex manifold $X$, then $k_{X, x}^{W, w}\left(x, x_{1}\right)>0$ for any invariant distance $d$ on any Riemann surface $W$.

Acknowledgements. It is my pleasure to thank Dr. Richard Timoney and Dr. David Wilkins for several helpful discussions and suggestions which refined the content of the original draft of this article. I would also like to thank Professor Seán Dineen for bringing J. P. Vigué's paper [4] to my attention. Finally I would like to acknowledge the referee's helpful observations.

\section{References}

[1] H. Cartan, Sur les fonctions de plusieurs variables complexes: l'itération des transformations intérieures d'un domaine borné, Math. Z. 35 (1932), 760-773. 
[2] J. M. LEE, "Riemannian manifolds. An introduction to curvature", Graduate Texts in Mathematics 176, Springer-Verlag, New York, 1997.

[3] W. Rinow, "Die innere Geometrie der metrischen Räume", Die Grundlehren der mathematischen Wissenschaften 105, SpringerVerlag, Berlin-Göttingen-Heidelberg, 1961.

[4] J. P. Vigué, Géodésiques complexes et rétractes holomorphes de dimension 1, Ann. Mat. Pura Appl. (4) 176 (1999), 95-112.

School of Mathematics

Trinity College Dublin

Dublin. 2

Ireland

E-mail address: colum@maths.tcd.ie

Primera versió rebuda el 14 de setembre de 2000, darrera versió rebuda el 2 d'octubre de 2001. 